\title{
Chroniques
}

génomiques

\section{Les débuts de CRISPR \\ en thérapie génique}

\section{Bertrand Jordan}

Depuis l'apparition de la technique CRISPR-Cas9, qui s'est répandue comme une traînée de poudre dans les laboratoires de recherche, son emploi en thérapie génique a souvent été évoqué [1]. L'entreprise Editas, une des plus actives dans ce secteur, s'intéresse notamment à l'amaurose congénitale de Leber $^{1}$, à d'autres pathologies rétiniennes, mais aussi à la mucoviscidose. Bien d'autres startup (CRISPR Therapeutics, Caribou Biosciences, Intellia) et au moins une Big Pharma (Novartis) se positionnent également dans ce domaine, souvent pour des applications concernant le cancer. En effet, bien que la thérapie génique soit souvent envisagée principalement dans le contexte des maladies génétiques, la majeure partie des essais cliniques en cours est en réalité centrée sur le traitement du cancer (Figure 1). Parmi ces essais, beaucoup concernent le prélèvement de lymphocytes $T$ du patient, leur modification pour les rendre plus actifs ou plus spécifiques, et leur multiplication au laboratoire avant réintroduction chez le malade [2]. C'est dans ce domaine que vont se dérouler les premiers essais cliniques utilisant la méthode CRISPR-Cas9. L'efficacité et la précision de cette méthode constituent en effet un avantage certain pour les interventions « chirurgicales » prévues sur ces cellules T puisqu'il s'agit d'introduire un changement très précis dans leur $A D N$, et si possible de modifier ainsi la majorité d'entre elles.

\section{Le projet américain}

Dirigé par Edward Stadtmauer à l'université de Pennsylvanie, il vise une amélioration du système déjà utilisé avec un certain succès par cette équipe. Dans un travail publié l'an dernier [3], ces chercheurs avaient, grâce à un vecteur de type lentivirus, introduit dans des cellules $T$ provenant des malades les séquences codant un récepteur T à haute affinité dirigé contre un antigène appelé NYESO-l et fréquemment exprimé par les cellules tumorales (il

${ }^{1}$ L'amaurose congénitale de Leber est une dystrophie et/ou une dysplasie rétinienne congénitale précoce. Elle constitue l'une des principales causes de cécité chez l'enfant. Elle est retrouvée chez environ 10 à $20 \%$ des enfants aveugles et représenterait $5 \%$ des dystrophies rétiniennes.

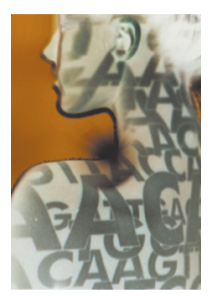

s'agissait alors de méla-
UMR 7268 ADÉS, Aix-Marseille, Université/ EFS/CNRS, Espace éthique méditerranéen, hôpital d'adultes la Timone, 264, rue Saint-Pierre, 13385 Marseille Cedex 05, France;

CoReBio PACA, case 901, parc scientifique de Luminy, 13288 Marseille Cedex 09, France. bertrand.jordan@univ-amu.fr brjordan@orange.fr obtenus étaient encourageants, avec une persistance des cellules $T$ modifiées durant plusieurs semaines après leur injection chez les malades, une réponse clinique nette (augmentation de la durée de survie sans progression ${ }^{2}$ ) et une toxicité modérée ${ }^{3}$. Le nouveau projet a pour but d'améliorer l'efficacité et la durée du traitement, en bloquant le principal mécanisme par lequel la tumeur «se défend » contre le système immunitaire de son hôte.

Rappelons que l'immuno-oncologie, après avoir connu des fortunes diverses au cours des dernières décennies, est actuellement en plein boom grâce à une meilleure compréhension des mécanismes par lesquels les tumeurs inhibent la réponse immunitaire, à l'identification des molécules de surface impliquées et à la mise au point d'anticorps monoclonaux qui, en bloquant ces molécules, suppriment l'inhibition, et permettent alors aux cellules $T$ de l'organisme de s'attaquer à la tumeur [4]. Un des systèmes les plus importants de ce point de vue est le couple formé par la protéine PD-1 (programmed cell death-1) exprimée sur les lymphocytes T et son ligand PD-Ll exprimé sur les cellules tumorales ou dans l'environnement immédiat de la tumeur. L'interaction entre PDl et PD-Ll inactive le lymphocyte T et favorise donc le développement de la tumeur. Un anticorps monoclonal dirigé contre PD-1, comme le Nivolumab (nom commercial Opdivo) peut rétablir la réponse immunitaire (Figure 2) et a donné des résultats spectaculaires pour

\footnotetext{
2 Par rapport à la durée habituelle : ce n'était pas un essai clinique comparatif en bonne et due forme.

${ }^{3}$ La description est sommaire, afin de ne pas alourdir le texte, et je passe sur diverses subtilités qui sont secondaires par rapport au message de cette chronique.
} 


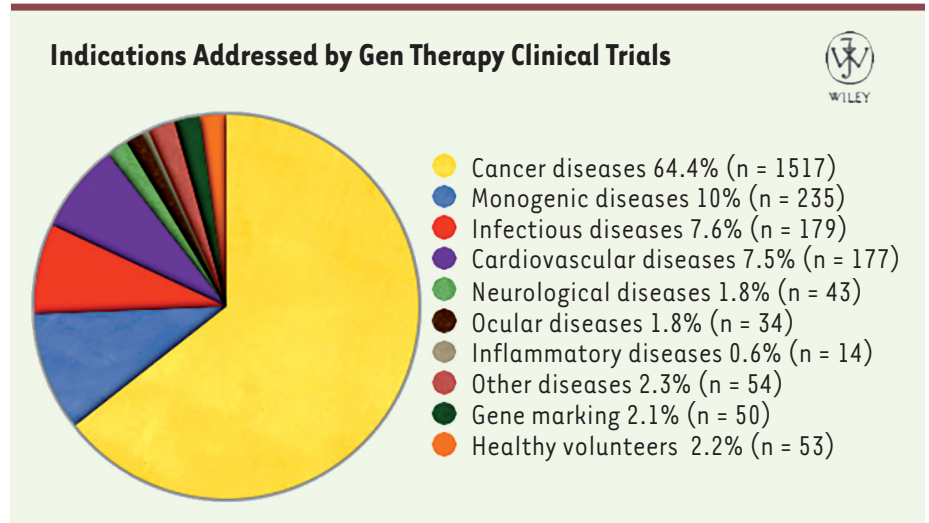

The Journal of Gene Medicine, ๔ John Wiley and Sons Ltd www.wiley.co.uk/genmed/clinical

Figure 1. Essais de thérapie génique par catégorie (http://www.abedia.com/wiley/).

le mélanome métastatique: pour certains patients, une survie prolongée (plusieurs années), peut-être même une guérison, alors que ce cancer est habituellement fatal à brève échéance. L'industrie pharmaceutique teste actuellement de nombreux inhibiteurs (anti-PD-1, anti-PD-L1, anti-CTLA-4 [cytotoxic T-lymphocyte associated protein 4$]^{4}$, etc.) sur de nombreux types de cancers, avec des résultats souvent prometteurs.

Le projet de Stadtmauer se propose d'améliorer le système T-NY-ESO-1 en inactivant, dans ces lymphocytes, le gène codant la protéine PD-1 : on peut espérer qu'alors les cellules $T$ introduites resteront actives bien plus longtemps, et que leur effet sera donc renforcé. C'est cette inactivation qui doit être réalisée par le système CRISPR-Cas9, en introduisant, dans les cellules déjà modifiées pour exprimer NY-ESO-1, les composants du système CRISPR-Cas9 avec un ARN guide (ARNg) ciblé sur l'exon 1 du gène PD-1. Après coupure de l'ADN en ce point par la nucléase Cas9, la réparation se fera par jonction non homologue (NHER, non-homologous end joining), inactivant ainsi le gène $P D-1$. En fait les auteurs comptent aussi introduire deux autres ARNg destinés à inactiver les gènes des chaînes alpha et bêta des récepteurs T préexistants dans la cellule, de manière à ce que le seul récepteur T exprimé soit celui qui est dirigé contre NY-ESO- $1^{5}$. Ils prévoient de traiter ainsi des malades présentant un sarcome, un myélome récidivant ou un mélanome, le but de ce premier essai étant de tester la faisabilité pratique de ces manipulations et la sécurité pour les patients.

Voilà donc un projet très ambitieux du point de vue technique puisqu'il repose sur une quadruple modification génique de cellules prélevées sur le patient, suivie de leur contrôle et de leur amplification in vitro puis de leur réintroduction. Et toutes ces manipulations devront être répétées pour chaque malade puisqu'il faut le traiter avec ses propres cellules. Notons que cela fait fort peu de temps que l'on sait utiliser le système CRISPRCas9 pour des lymphocytes $T$ : les premières démonstrations datent d'un an à peine $[5,6]$. Comme l'efficacité de la correction du génome (genome editing) n'est pas de $100 \%$, et que les trois modifications introduites sont

\footnotetext{
${ }^{4}$ CTLA-4 est un régulateur négatif de la réponse T. II entre en compétition avec le co-récepteur activateur CD28, vis-à-vis de CD80 et CD86.

${ }^{5}$ En fait, contre un peptide dérivé de la protéine NY-ESO-1 présentée dans le contexte HLA-A2 (human leukocyte antigens) de la cellule. On ne peut donc utiliser cette approche que sur des patients possédant cet allèle, qui est heureusement très fréquent.
}

indépendantes, il est probable que la proportion de cellules T portant l'ensemble des changements désirés sera faible ${ }^{6}$. II faudra bien, d'autre part, vérifier l'absence de modifications survenues ailleurs dans le génome (off-target). Le projet a été examiné par le comité RAC (Recombinant DNA Advisory Committee) des NIH (National Institutes of Health), qui est l'instance compétente aux États-Unis pour les essais de thérapie génique ; après un débat que l'on peut consulter in extenso sur internet [7], il a été approuvé à l'unanimité moins une abstention. II reste à obtenir l'accord du comité de l'Université et celui de la FDA (Federal Drug Administration), le traitement étant considéré comme un nouveau médicament, mais a priori l'accord du RAC est le plus important et devrait entraîner l'adhésion des autres instances. II est donc probable que cet essai puisse débuter d'ici la fin de l'année 2016.

\section{La surprise chinoise}

Mais voilà que, le 21 juillet 2016, un communiqué émanant de l'université du Sichuan annonçait le début imminent (en août) d'un essai a priori comparable [8]. Déjà approuvé par le comité d'éthique de l'hôpital (West China Hospital) dépendant de cette université, il est présenté comme un essai de phase I visant à vérifier la sécurité du traitement mais aussi à évaluer son efficacité et le taux de réponse. II sera pratiqué sur les patients atteints de cancer du poumon avancé (NSCLC, non-small cell lung cancer), et comportera le prélèvement de lymphocytes T périphériques, leur modification par CRISPR-Cas9 afin d'inactiver le gène PD-1, et leur multiplication avant réintroduction chez le malade. Avant injection des cellules T modifiées, les patients seront, comme d'ailleurs dans le projet américain, traités par chimiothérapie pour réduire leur population de cellules $T$; ils recevront de plus de l'interleukine 2 après l'injection afin d'aider à la multiplication des cellules $T$ introduites. Une entreprise locale appelée Chengdu MedGenCell se chargera des contrôles sur les cellules avant injection. L'essai proprement dit est prévu pour durer six mois, les résultats finaux étant attendus pour début 2018. II est dûment enregistré dans la base de données américaine Clinical Trials [9]. Cet essai apparaît moins sophistiqué que le précédent, puisqu'il se borne à inactiver le gène $\mathrm{PD}-1$ dans les lymphocytes $T$ « tout venant », sans chercher à les cibler sur un antigène exprimé dans la tumeur: par ailleurs il s'adresse à un type de cancer (NSCLC) pour lequel les immunothérapies n'ont jusqu'ici pas montré une très forte efficacité (au contraire du mélanome). Cela dit, il ne semble pas absurde de commencer par un protocole relativement simple (inactivation

${ }^{6}$ Si l'efficacité de chaque changement était de $30 \%$ (ce qui est déjà un très bon chiffre), moins de $3 \%$ des cellules seraient triplement modifiées 


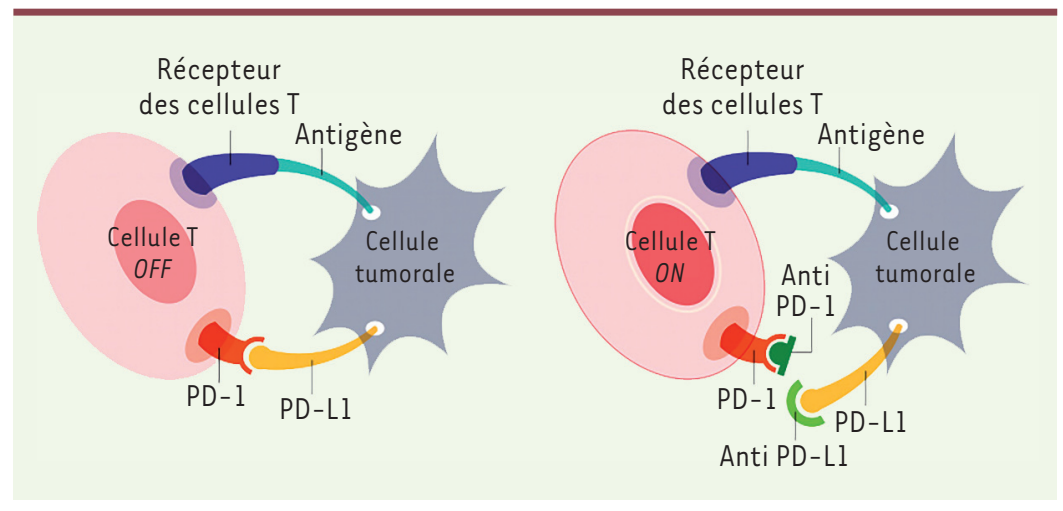

Figure 2. Schéma (très simplifié) de l'inactivation d'une cellule $T$ (à gauche) via l'interaction entre PD-1 (programmed cell death 1 ) et son ligand (PDL1) exprimé sur la cellule tumorale, et de sa réactivation (à droite) grâce à un anticorps anti-PD-1 ou anti-PD-L1. Le récepteur des cellules $T$ reconnaît en fait un peptide dérivé de l'antigène tumoral et présenté par une molécule HLA (ou CMH, complexe majeur d'histocompatibilité) (non représentée) (ㄷ) image de Smartpatients.com, Creative Commons).

du seul gène $P D-1$ ) afin d'explorer cette voie; le cancer du poumon est bien plus fréquent que le mélanome métastatique, et pour cet essai le typage HLA du patient n'a pas d'importance - autant de facteurs qui doivent permettre un recrutement rapide. En revanche, les cellules T modifiées n'étant pas ciblées sur la tumeur (contrairement à l'essai américain), elles risquent de s'attaquer à d'autres tissus et de provoquer des effets auto-immuns sévères [10].

\section{Recherche médicale et géopolitique}

À propos de cet essai chinois, dont l'annonce a pris le monde scientifique par surprise [8], on ne peut que noter la célérité et la simplicité des procédures d'approbation : il a apparemment suffi de l'accord d'un comité local, celui de l'hôpital au sein duquel est réalisé l'essai. On peut légitimement se demander si cet examen a été réalisé avec toute l'indépendance et la rigueur souhaitées, d'autant plus que l'on peut craindre des effets secondaires liés à des problèmes d'auto-immunité. II est clair que des facteurs extrascientifiques ont joué : la Chine cherche à apparaître comme une nation pionnière pour les applications de la technique CRISPR. Elle se prévaut déjà d'avoir obtenu les premiers primates modifiés par CRISPR [11] et bien sûr - nous nous en sommes déjà fait l'écho - les premiers embryons humains (non viables) ayant subi une telle opération [12] : l'espoir de faire aussi la démonstration de la première application thérapeutique de la technique a sans doute joué un rôle dans cette affaire, d'autant plus que la culture chinoise en matière d'éthique de la recherche est assez différente de la nôtre [13]. Espérons simplement que les malades participant à l'essai n'en feront pas les frais... $\diamond$

\section{SUMMARY}

\section{First use of CRISPR for gene therapy}

Two clinical trials using CRISPR technology to engineer the $T$ cells of cancer patients by inactivating the gene for immune checkpoint molecule PD-1 are (almost) underway, one in the USA and the other in China. The less sophisticated Chinese trial seems slated to begin first, in part because of very speedy approval ; it may however trigger autoimmune reactions that could be very serious. $\diamond$

\section{LIENS D'INTÉRÊT}

L'auteur déclare n'avoir aucun lien d'intérêt concernant les données publiées dans cet article.

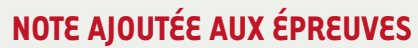

Dernière minute : le 15 novembre, les responsables de l'essai chinois [8] ont indiqué avoir traité leur premier patient le 28 octobre. Cette communication inhabituelle confirme bien les enjeux politiques de cet essai. Nature 15 novembre 2016 (http://www.nature.com/news/crispr-geneediting-tested-in-a-person-for-the-first-time-1.20988).

\section{RÉFÉRENCES}

1. Jordan B. CRISPR-Cas9, une nouvelle donne pour la thérapie génique. Med Sci (Paris) $2015 ; 31: 1035-8$.

2. Fesnak AD, June CH, Levine BL. Engineered T cells: the promise and challenges of cancer immunotherapy. Nat Rev Cancer 2016; 16 : 566-81.

3. Rapoport AP, Stadtmauer EA, Binder-Scholl GK, et al. NY-ESO-1-specific TCRengineered T cells mediate sustained antigen-specific antitumor effects in myeloma. Nat Med $2015 ; 21$ : 914-21.

4. Kreamer KM. Immune checkpoint blockade: a new paradigm in treating advanced cancer. J Adv Pract Oncol 2014 ; 5 : 418-31.

5. Schumann K, Lin S, Boyer $\varepsilon$, et al. Generation of knock-in primary human T cells using Cas9 ribonucleoproteins. Proc Natl Acad Sci USA 2015 ; 112 : 10437-42.

6. Su S, Hu B, Shao J CRISPR-Cas9 mediated efficient PD-1 disruption on human primary T cells from cancer patients. Sci Rep 2016 ; 6 : 20070.

7. https://fr.scribd.com/doc/316280454/RAC-Review-Penn-Medicine-CRISPRNYESO-1-Immunetherapy-study (Accessed 28/8/2016)

8. Cyranoski D. Chinese scientists to pioneer first human CRISPR trial. Nature 2016 ; $535: 476-7$.

9. https://clinicaltrials.gov/ct2/show/NCT02793856 (accessed 28/8/2016).

10. Champiat S, Lambotte 0 , Barreau $\varepsilon$, et al. Management of immune checkpoint blockade dysimmune toxicities: a collaborative position paper. Ann Oncol 2016 ; $27: 559-74$.

11. Niu Y, Shen B, Cui Y, et al. Generation of gene-modified cynomolgus monkey via Cas9/RNA-mediated gene targeting in one-cell embryos. Cell $2014 ; 156: 836-43$.

12. Liang $P, X u Y$, Zhang $X$, et al. CRISPR/Cas9-mediated gene editing in human tripronuclear zygotes. Protein Cell $2015 ; 6: 363-72$.

13. Peng $Y$. The morality and ethics governing CRISPR-Cas9 patents in China. Nat Biotechnol $2016 ; 34: 616-8$.

\section{TIRÉS À PART}

B. Jordan 